\title{
DETECTION OF NORMALITY/PATHOLOGY ON CHEST RADIOGRAPHS USING LBP
}

\author{
Juan Manuel Carrillo-de-Gea, Ginés García-Mateos \\ Dept. Informática y Sistemas, Faculty of Computer Science, University of Murcia, Spain \\ jmcdg1@um.es,ginesgm@um.es
}

Keywords: Image processing, health informatics, X-rays, computer-aided detection (CADe), template matching, local binary patterns (LBP), histograms.

\begin{abstract}
Since the discovery of X-rays and their applications, medical imaging has been a great help for radiologists in the diagnosis of diseases. In recent years, there has been a great effort in the computer vision community in the research of systems for the analysis and extraction of useful information from medical images. In this scenario, we have designed, implemented and validated a novel method to detect normality/pathology in chest radiographs, which constitutes the core of a computer-aided detection (CADe) system. Although the problem addressed is very complex and little explored, our approach is completely automatic, starting from the location of areas of interest using template matching techniques. The main novelty of our contribution is the application of a transformation known as local binary patterns (LBP) to these areas. LBP histograms are then used as input features for a classification system, which is ultimately responsible for the decision of normality/pathology. The results of our preliminary experiments are quite promising. With success rates in the best cases close to $90 \%$, we believe that increased performance could be obtained with bigger training sets and more advanced classification systems, which will make these systems to be fully viable in the near future.
\end{abstract}

\section{INTRODUCTION}

The diagnostic imaging is based on the use of different physical principles to visualize the internal structure, composition and functions of living organisms, so that medical professionals have evidence available to identify lesions in patients. Among the various existing medical imaging techniques, we can name a few: based on X-rays (including conventional radiography), based on nuclear magnetic resonance, based on the reflection of ultrasound and based on emissions from radioisotopes (Fleckenstein and Tranum-Jensen, 2001). Figure 1 shows a sample of a chest radiograph.

Despite the subsequent emergence of new imaging techniques, nowadays conventional radiology remains to have a central role in health care facilities around the world, "due to its ability of revealing some unsuspected pathologic alterations, its non-invasivity characteristics, radiation dose and economic considerations" (Campadelli and Casiraghi, 2005, p. 1431).

Also, computer systems have been widely used

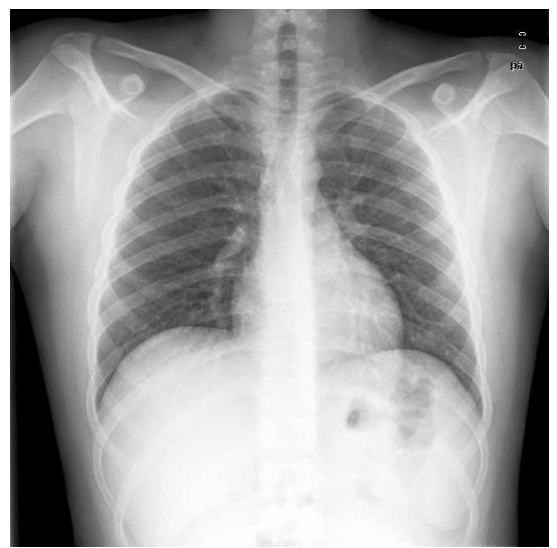

Figure 1: Male chest radiograph in posteroanterior (from back to front) view.

in the analysis of medical signals. Over the past 15 years many research groups have focused on the development of computerized systems that can examine 
different types of medical images and extract useful information for medical professionals (Papadopoulos et al., 2005), specifically assisting in image analysis and diagnostic decision making. Such systems are commonly referred to as computer-aided detection/diagnosis (CAD) systems.

As we will review in section 2, there is an interesting amount of work done in this field. However, our proposal represents a new point of view, since it is a method aimed to detect normality and pathology from posteroanterior (PA) chest radiographs instead of being specialized in a predefined number of diseases in specific organs of the body. We should note that the problem addressed is a little explored field, and more complex than most of the existing computeraided detection (CAD detection) systems.

The rest of the paper is organized as follows. In section 2, we present a classification of CAD systems and review some approaches. In section 3, the proposed method is described in detail, and in section 4 we expose and discuss the results of performed tests. Finally, conclusions are presented in section 5.

\section{RELATED WORK}

\subsection{Classification of CAD Systems}

According to (Kallergi, 2005; Papadopoulos et al., 2005), computer-aided biomedical systems can be classified into the first two categories of the list below. More recently, a third type of system has been incorporated (Hao et al., 2008):

- Computer-aided detection (CAD detection or CADe) systems, which detect signs of pathology in medical images by extracting features that are highly correlated with the type and nature of the abnormality under investigation, but do not provide a medical diagnosis as such.

- Computer-aided diagnosis (CAD diagnosis or CADx) systems, which assist clinicians in diagnosis. Based on the same or additional features, such schemes characterize the identified regions according to their pathologies.

- Computer-aided change detection (CADcd) systems, which provide detection and diagnosis as well as evaluation of the progression of the disease by identifying the areas that have changed.

\subsection{Review of Existing CAD Systems}

CAD systems have attracted the interest of the computer vision community in the last decade. There is an extensive literature in the domain of breast and lung cancer imaging. Actually, there are techniques related to the study of CAD systems ready to detect other diseases such as severe acute respiratory syndrome (SARS), acute intracranial haemorrhage (AIH), colon polyps and thyroid gland nodules. We can even find research works focused on the use of imaging techniques different from x-rays, such as ultrasound.

On the other hand, the local binary pattern (LBP) operator is introduced as a way to summarize the structure of local gray levels of an image (Ojala et al., 1996). Regarding the application of LBP or its variations to solve medical imaging problems, researchers are getting very good results, often improving other existing approaches (Iakovidis et al., 2008; Oliver et al., 2007; Sørensen et al., 2008).

There are very few works dealing with the problem of normality and pathology detection without specializing in certain diseases. Research done by (Park et al., 2004), aimed to the detection of normal/abnormal texture on chest radiographs, represents an exception. However, they propose the utilization of techniques different from ours, such as a knowledge based lung field extraction method, filtering to reduce ribs shadows, feature extraction applying a cuasi-Gabor filter in the frequency domain and a k-nearest-neighbors classifier. Moreover, in (Sørensen et al., 2008), authors try to solve the problem of texture classification in lung using LBP. However, in this research work, computed tomography images are used instead of conventional radiographs. LBP histogram similarity is used as distance measure. Then, classification is performed using the k-nearestneighbor classifier with these distances as input.

\section{OUR APPROACH USING LBP}

In this section, we describe our CADe system specialized in PA chest radiographs. According to (Papadopoulos et al., 2005), the main phases of a typical CAD detection system are: preprocessing, segmentation, feature analysis (extraction, selection and validation), and classification, used to reduce false positives or to characterize abnormalities. Figure 2 represents the structure of this kind of systems.

\subsection{Digital Radiography}

The input of our system is a set of DICOM (Digital Imaging and COmmunications in Medicine) digital radiological images. The images are around $3000 \times 3000$ pixels with 12 bits per pixel. DICOM is a well-known standard for exchanging medical images, 


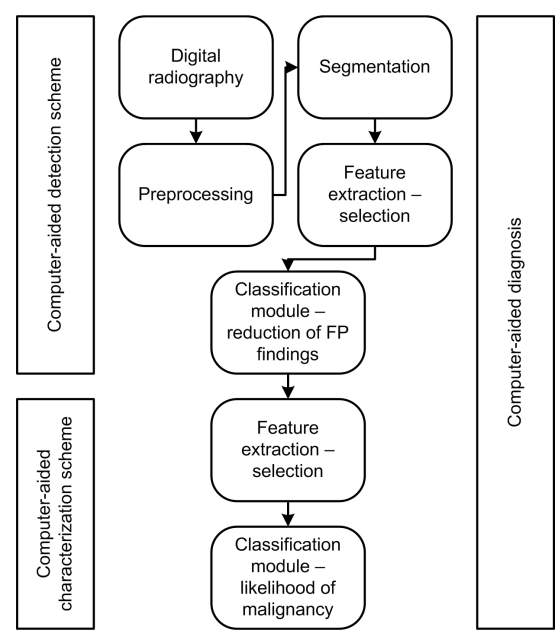

Figure 2: CAD architecture, (Papadopoulos et al., 2005).

designed for handling, storage, printing and transmission of medical images.

\subsection{Preprocessing}

We first transform the digital radiographs into a format more suitable for easier processing, using MRIcro $^{1}$, which is a freeware tool for efficient viewing and exporting of medical images. We chose the PNG (Portable Network Graphics) format, which is based on a lossless compression algorithm. This step implies the reduction of DICOM image depth from 12 bits per pixel to 8 bits per pixel; however, this more compact representation of the image data still keeps the information necessary for our purposes.

By using LBP, the necessity of applying image equalization, or other preprocessing steps, is removed. As we will describe in section 3.4, in LBP images only the value of a pixel in relation to its neighbors is relevant. Nevertheless, since our system input are high resolution radiographs, the needs of time and memory could be too demanding. Therefore, before the segmentation stage we apply a decimation to the input images done by super-sampling interpolation.

\subsection{Segmentation}

In this stage, the original image is segregated into separate parts. As in our system the main object of interest are the lungs, the goal of segmentation is to determine the position and size of both lungs.

There are a variety of techniques that can be used for segmentation. Our proposal is based on template

\footnotetext{
${ }^{1}$ http://www.sph.sc.edu/comd/rorden/mricro.html
}

matching, a technique for finding those parts of an image which match a predefined template image.

\subsubsection{Proposed Segmentation Method}

We utilize patterns of the right and left lungs as input for a template matching algorithm. It is important to use different patterns of lungs, to include the wide variety of appearances that may be caused by sex, age of individuals and their archetype. Furthermore, a multiscale search allow us to locate lungs with different sizes. The measure used for matching is a normalized correlation coefficient (NCC).

The point of maximum correlation, which is determined by the map of results produced by the template matching algorithm, indicates the position of the lungs. The dimensions are those corresponding to the chosen pattern at the scale selected.

Finally, according to the obtained locations, the lungs are divided into regions of equal size (see figure 3 ). This division is required in the subsequent steps.

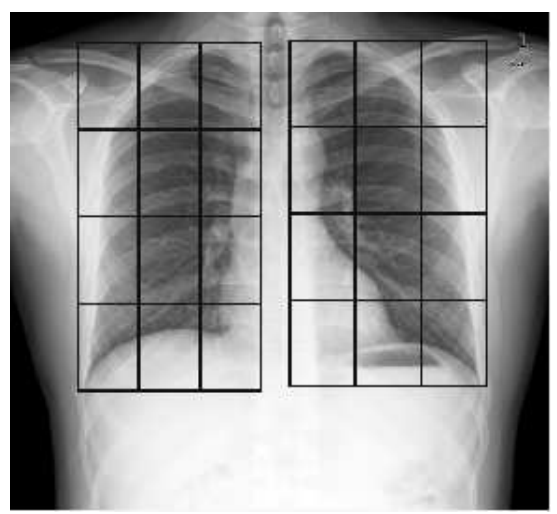

Figure 3: Localization of lungs in a radiograph and segmentation of them into regions. In the experiments described on section 4 , we have used a grid of $3 \times 4$ rectangular regions.

\subsection{Feature Extraction}

Feature extraction consists on the calculation of some image features, looking for a description of the properties of each object. Our proposal is to use LBP as the basic method to extract texture features in the images (Ojala et al., 1996). This is a gray-scale invariant texture measure, derived from a general definition of texture in a local neighborhood. Although this technique has been used in other medical imaging applications, this work represents, to the best of our knowledge, the first application to test of normality/pathology on radiographs.

The LBP operator was originally defined for $3 \times 3$ neighborhoods, giving 8 bit codes based on the 8 pi- 
xels around the central pixel. The original LBP operator labels all the pixels of the image by thresholding the neighborhood of each pixel with the central value and considering the result as a binary number. A sample of this calculation is shown in figure 4.

\begin{tabular}{|l|l|l|}
\hline 50 & 24 & 76 \\
\hline 18 & 34 & 19 \\
\hline 34 & 87 & 45 \\
\hline
\end{tabular}

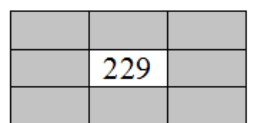

Threshold $\square$

\begin{tabular}{|l|l|l|}
\hline 1 & 0 & 1 \\
\hline 0 & & 0 \\
\hline 1 & 1 & 1 \\
\hline
\end{tabular}

U Addition
\begin{tabular}{|c|c|c|}
\hline 1 & 0 & 4 \\
\hline 0 & & 0 \\
\hline 32 & 64 & 128 \\
\hline
\end{tabular}

\begin{tabular}{|c|c|c|} 
\& \\
\hline 1 & 2 & 4 \\
\hline 8 & & 16 \\
\hline 32 & 64 & 128 \\
\hline
\end{tabular}

Multiplication

Figure 4: Example of a LBP calculation.

The histogram of these $2^{8}=256$ different labels can then be used as a texture descriptor. The local primitives encoded by these cells include different types of curved edges, spots, flat areas, etc. The calculation of the LBP codes can be easily done in a single pass through the image. Formally, the LBP operator takes the form shown in (1), where $n$ iterates on the 8 neighbors of the central pixel $c, g_{c}$ and $g_{n}$ are the grayscale level values of $c$ and $n$ respectively, and $s$ defines the thresholding function shown in equation (2).

$$
\begin{gathered}
\operatorname{LBP}\left(x_{c}, y_{c}\right)=\sum_{n=0}^{7} 2^{n} s\left(g_{n}-g_{c}\right) \\
s(u)= \begin{cases}0, & u \geq 0 \\
1, & u<0\end{cases}
\end{gathered}
$$

\subsubsection{Proposed Feature Extraction Method}

We apply LBP technique going over each pixel in the radiograph and accessing to its $3 \times 3$ pixel vicinity. A typical application to a chest radiograph is shown in figure 5. Next, the LBP image is analyzed by regions (see figure 6), computing the histograms of each region of the lungs (as previously found). Then we normalize the histograms for comparison.

\subsection{Classification}

The diagnosis is an integrated medical procedure aimed to the identification of a disease from its signs and symptoms. In the same way, the classification stage is an essential and critical part of a CAD system. Classification methods range from techniques

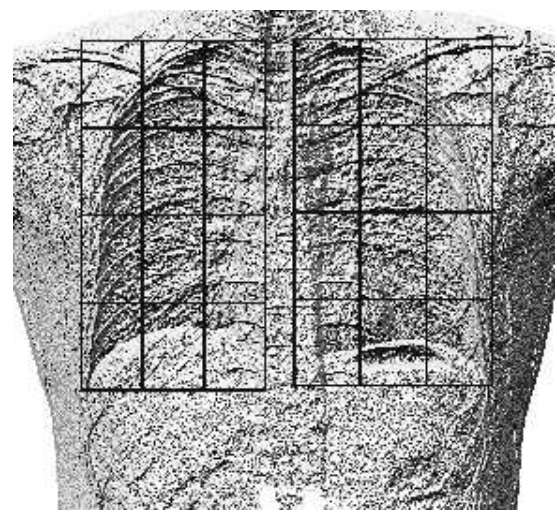

Figure 5: Application of LBP operator to a radiograph.

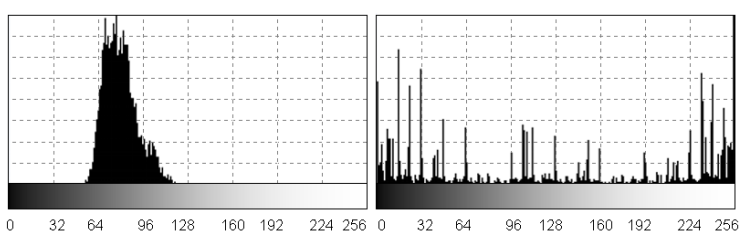

Figure 6: Comparison between the histogram of gray levels and the LBP histogram calculated for the same region.

that classify lesions according to their types, to techniques that produce binary diagnostics, as is our case, since the objective is to determine whether the radiograph is normal or contains some kind of pathology.

Despite the existence of a wide variety of complex classifiers for analyzing medical images, since we are limited by the number of training examples, we propose to use conventional classifiers.

\subsubsection{Proposed Classification Method}

The main stages of our approach are:

- Calculation of distances between histograms, which consists of obtaining distances between histograms of each region of the lungs of the given radiograph and those histograms corresponding in normal and pathologic radiographs of the training set. At the same time, minimum distances to normal and pathologic radiographs are obtained for each region. We use the Bhattacharyya distance for comparison between two histograms.

- Differences between minimum distances and classification of regions. For each region, the difference between the minimum distance to normal and pathologic histograms is obtained. The values obtained indicate whether a histogram is closer to one class or another (to normal or patho$\operatorname{logy})$. Apart from calculating the differences between minimum distances, we check the sign of 
these differences and classify the regions as they are closer to the corresponding region of the normal or the pathologic images.

- Classification of radiographs with or without information on discrimination of regions, which is aimed at the final classification of the radiograph. We have analyzed three different classification methods, which will be compared in the experiments: greater difference in absolute value (GDAV), discrete voting (DV) and continuous voting (CV). GDAV method consists on going through all regions of the lungs looking for the difference between the minimum distances (as calculated above) that is larger in absolute value. Once obtained, the class of the radiograph is the same as that of the region concerned. DV method is a voting in which each region, depending on its class, contributes with one positive or negative vote. When all the votes are collected, if the number exceeds a certain threshold the image is considered as normal and otherwise as pathologic. CV method is similar to DV method, but now there is a global sum reflecting the accumulation of the differences between the minimum distances of the input image to the normal and to the pathologic images, for each region of the lungs. At the end, if the sum exceeds a certain threshold the image is considered as pathologic and otherwise as normal.

Note that the threshold established for voting methods is determinant to control the compromise between false positives (FP) and false negatives (FN). In turn, the three methods have two variants: using discrimination matrix or not. This matrix weights the relative importance of each region of the grids in which the lungs are divided. When no discrimination matrix is used, we have no information about the level of discrimination of each region for the determination of normality and pathology, so it is assumed that all the regions of the grid have exactly the same importance. The discrimination matrix is obtained automatically through training with the same set of normal and pathologic radiographs available.

\section{EXPERIMENTAL RESULTS}

The clinical performance of medical tests, including medical imaging, is normally determined by estimating indexes for true positives (TP), true negatives (TN), false positives (FP), false negatives (FP), sensitivity, specificity and accuracy, among others (Kallergi, 2005). In addition, confusion matrices as the one shown in table 1 are often used.
Table 1: Confusion matrix.

\begin{tabular}{|l|l|l|l|}
\cline { 3 - 4 } \multicolumn{2}{c|}{} & \multicolumn{2}{|c|}{ Prediction Outcome } \\
\cline { 3 - 4 } \multicolumn{2}{c|}{} & Normal & Pathologic \\
\hline Actual & Normal & TN & FP \\
\cline { 2 - 4 } & Pathologic & FN & TP \\
\hline
\end{tabular}

We have 25 normal (12 males and 13 females) and 23 pathologic (13 males and 10 females) high resolution DICOM images of chest radiographs to perform tests. With this input, we have conducted an extensive battery of tests using the leave-one-out technique.

In each test, we measured the following values: sensitivity $S E N S=\frac{T P}{T P+F N}$ (probability of giving a correct response to the pathologic cases), specificity $S P E C=\frac{T N}{T N+F P}$ (probability of obtaining a correct response to the cases with no pathology) and $a c c u$ racy $A C C=\frac{T P+T N}{T P+T N+F P+F N}$ (equivalent to the proportion of successes over the total).

The experimental tests performed have produced the results summarized in table 2 .

Table 2: Results of the tests using the GDAV, DV and CV classifiers (in this order). WOD and WD means without discrimination and with discrimination matrix, respectively. $\mathrm{M}, \mathrm{F}$ and $\mathrm{A}$ means using male, female and all radiographs, respectively.

\begin{tabular}{|c|c|c|c|c|c|c|}
\cline { 2 - 7 } \multicolumn{1}{c|}{} & \multicolumn{3}{c|}{ WOD } & \multicolumn{3}{c|}{ WD } \\
\cline { 2 - 7 } \multicolumn{1}{c|}{} & M & F & A & M & F & A \\
\hline SENS & 0.31 & 0.4 & 0.39 & 0.54 & 0.4 & 0.39 \\
\hline SPEC & 0.67 & 0.85 & 0.96 & 0.83 & 1 & 0.88 \\
\hline ACC & 0.48 & 0.65 & 0.69 & 0.68 & $\mathbf{0 . 7 4}$ & 0.65 \\
\hline \hline SENS & 0.46 & 0.6 & 0.96 & 0.92 & 0.7 & 0.78 \\
\hline SPEC & 0.67 & 0.77 & 0.2 & 0.5 & 0.77 & 0.8 \\
\hline ACC & 0.56 & 0.69 & 0.56 & 0.72 & 0.74 & $\mathbf{0 . 7 9}$ \\
\hline \hline SENS & 0.46 & 0.7 & 0.7 & 0.54 & 0.7 & 0.52 \\
\hline SPEC & 0.67 & 0.54 & 0.44 & 0.75 & 1 & 0.88 \\
\hline ACC & 0.56 & 0.61 & 0.56 & 0.64 & $\mathbf{0 . 8 7}$ & 0.71 \\
\hline
\end{tabular}

\subsection{Discussion of the Results}

Given the considerable amount of data available, we will focus our attention on the accuracy obtained in each of the experiments. We will analyze and discuss methods that do not use discrimination matrix and those that use it.

For the methods without discrimination information, there is a general low level of precision, as shown in figure 7 . This is more pronounced in the voting methods. GDAV method has an interesting accuracy rate, especially for female radiographs and when the radiographs are considered all together. Male radio- 
graphs are the most difficult to classify in this set of data, but we believe that this fact is not generalizable. Finally, although there are some cases with less than $50 \%$ accuracy (on male radiographs), the average accuracy is around $55 \%$ with peaks close to $70 \%$.

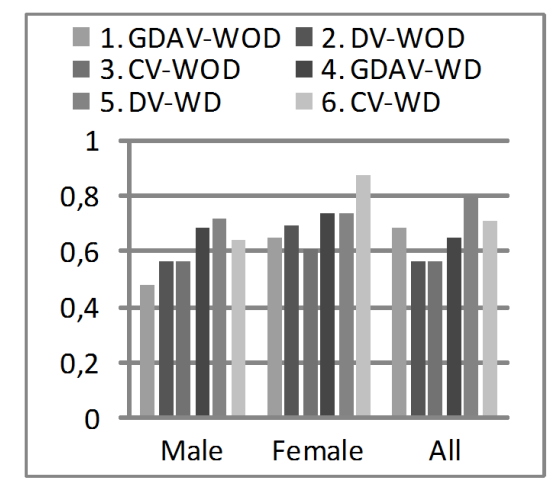

Figure 7: Accuracy obtained with different classifiers with and without using discrimination matrix.

Regarding discrimination methods, the degree of accuracy achieved is clearly better, highlighting the classification of female radiographs again. In most cases, voting methods perform better than GDAV method. It is remarkable that with the appropriate threshold, the CV method classifies women radiographs with success rates very close to $90 \%$ of accuracy. With all the radiographs the best is DV method, reaching an accuracy near to $80 \%$. In general, the average accuracy is around $70 \%$ considering all cases.

\section{CONCLUSIONS}

As a main general conclusion, the utilization of the LBP texture analysis operator has emerged as an effective means for the study of radiographic images, and more particularly chest radiographs, which are the focus of this paper. LBP is a method that collects interesting advantages as a small number of features and a high power of discrimination.

The use of template matching technique at the stage of segmentation to locate the lungs has been satisfactory, despite its known limitations. The great variability among individuals in terms of shape and size of the lungs makes it difficult to achieve an optimal location of these structures.

Our approach to the problem has been implemented and tested as a first step towards a system for the detection of normality and pathology in radiographs which can be implanted in a hospital. The ratios of success achieved, near $90 \%$ in the best cases, are quite promising, although the method designed should not be considered closed. Clearly, higher accuracy rates should to be obtained to achieve a practical CAD system. To work in its development the use of much larger sets of test will be essential.

\section{ACKNOWLEDGEMENTS}

This work has been supported by the Spanish MEC and European FEDER grants TIN2006-15516-C0403 and Consolider CSD2006-00046.

\section{REFERENCES}

Campadelli, P. and Casiraghi, E. (2005). Lung Field Segmentation in Digital Postero-Anterior Chest Radiographs. In PRIA, volume 3687 of LNCS, pages 736745. Springer Heidelberg.

Fleckenstein, P. and Tranum-Jensen, J. (2001). Anatomy in Diagnostic Imaging. Blackwell Science, 2 edition.

Hao, B., Leung, C. K.-S., Camorlinga, S., Reed, M. H., Bunge, M. K., Wrogemann, J., and Higgins, R. J. (2008). A Computer-Aided Change Detection System for Paediatric Acute Intracranial Haemorrhage. In C3S2E '08, pages 109-111, New York. ACM.

Iakovidis, D. K., Keramidas, E. G., and Maroulis, D. (2008). Fuzzy Local Binary Patterns for Ultrasound Texture Characterization. In IAR, volume 5112 of LNCS, pages 750-759. Springer Heidelberg.

Kallergi, M. (2005). Evaluation Strategies for MedicalImage Analysis and Processing Metodologies. In $\mathrm{Me}$ dical Image Analysis Methods, chapter 12, pages 433471. CRC Press.

Ojala, T., Pietikäinen, M., and Harwood, D. (1996). A Comparative Study of Texture Measures with Classification Based on Feature Distributions. Pattern Recognition, 29(1):51-59.

Oliver, A., Llad, X., Freixenet, J., and Mart, J. (2007). False Positive Reduction in Mammographic Mass Detection Using Local Binary Patterns. In MICCAI '07, volume 4791 of $L N C S$, pages 286-293. Springer Heidelberg.

Papadopoulos, A. N., Plissiti, M. E., and Fotiadis, D. I. (2005). Medical-Image Processing and Analysis for CAD Systems. In Medical Image Analysis Methods, The Electrical Engineering and Applied Signal Processing Series, chapter 2, pages 51-86. CRC Press, Boca Raton, FL, USA

Park, M., Jin, J. S., and Wilson, L. S. (2004). Detection of Abnormal Texture in Chest X-rays with Reduction of Ribs. In VIP '05, pages 71-74, Darlinghurst, Australia. Australian Computer Society, Inc.

Sørensen, L., Shaker, S. B., and de Bruijne, M. (2008). Texture Classification in Lung CT Using Local Binary Patterns. In MICCAI '08, volume 5241 of LNCS, pages 934-941. Springer Heidelberg. 